\title{
Computer Aided Symbolic Linear Algebra
}

\author{
Juan M. de Olazábal \\ Universidad de Cantabria, Dpto. de Matemáticas 39005 Santander , SPAIN \\ jm.olazabal@unican.es \\ http://www.matesco.unican.es/\%7Eolazabal
}

\begin{abstract}
The aim of this communication is to present a Symbolic Linear Algebra package, written in MAPLE V.5 and working also in the release 7 , with general but especially educational purpose. Its goal is to run over MAPLE the different Linear Algebra algorithms developped in [OJ]. The implemented algorithms are also valids over finite characteristic and, for educational purpose, may be runned interactively. Three topics are covered by the package: equivalent matrices (echelon matrices, rank, LU decomposition, linear systems,...), similar matrices (Eigenvalues and eigenvectors, Rational or Frobenius form, Irreducible form, Jordan form,...), and congruent matrices (Symmetric and Hermitian matrices, Cholesky and QR decomposition, SVD, Gram-Schmidt over arbitrary euclidean metrics, ortogonal matrices, quadric surfaces...)
\end{abstract}

\section{Introduction}

Maple is an excellent computer algebra system of general purpose, allowing user to write procedures in order to build different packages on his own. In this way and for educational purpose, we have "packaged" several procedures with additional possibilities to classic Maple jobs.

For instance, as it is well known, function $\operatorname{rank}(\mathrm{A})$ computes the rank of the matrix A; so if you consider the matrix

$$
A=\left(\begin{array}{lll}
t & 1 & 1 \\
1 & t & 1 \\
1 & 1 & t
\end{array}\right)
$$

$>\operatorname{linalg}[\mathrm{rank}](\mathrm{A})$;

computes over the field $\mathbf{Q}(\mathrm{t})$ and its output is 3 .

But if you prefers to compute, as it is usual in the first courses of Linear Algebra, the different values of the rank when $t$ runs over rationals, you may use the package CASLA, presented in this communication; it has a upper-cased function RANK which allows different parameters.

In this way, $\operatorname{RANK}(\mathrm{A}, \mathrm{t})$ produces the output in figure 1

Moreover, from an educational point of view, if the user wants to learn the computation of the rank of any matrix, function linalg[rank] does not help him.

By passing the argument <inter > to upper-case function RANK, we have the interactive job in figure 2 . 


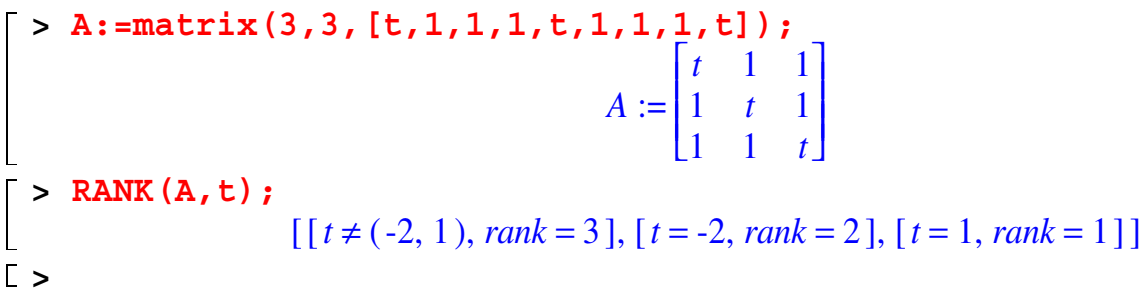

Fig. 1. RANK of parametric matrices

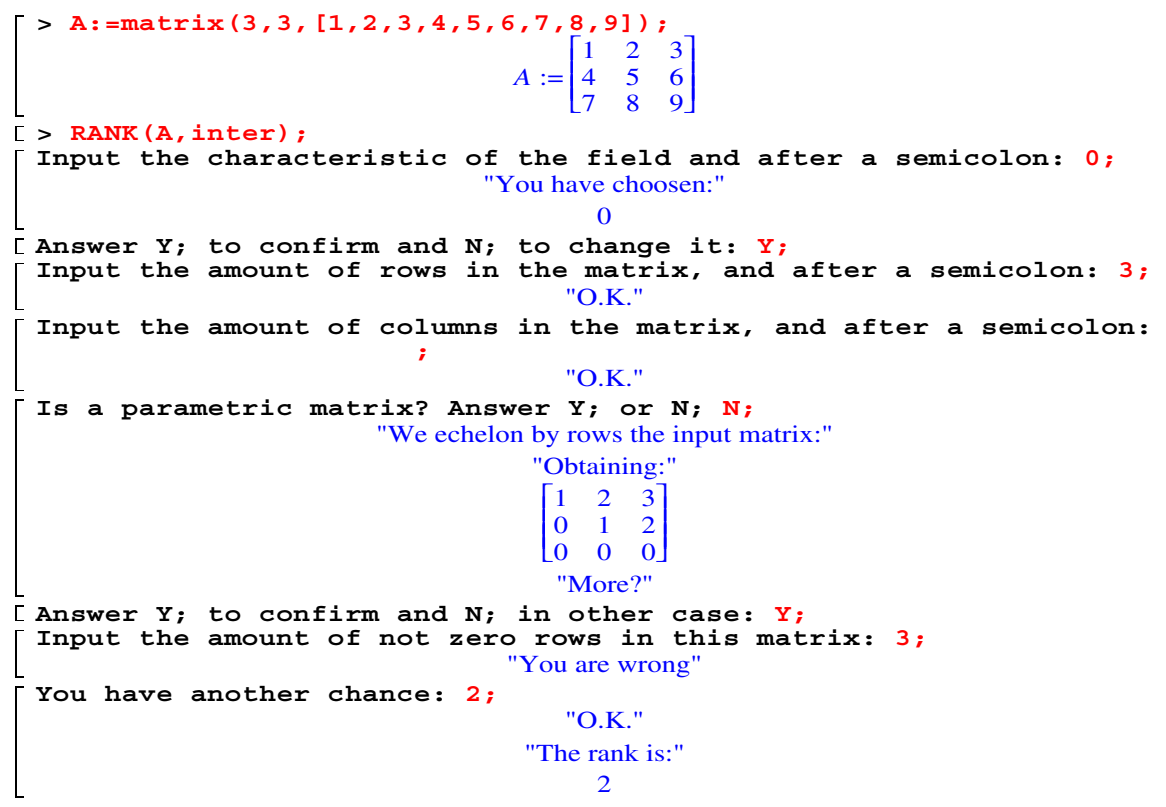

Fig. 2. Interactive job of RANK

Most functions of this package allow the reserved parameter inter. When functions are called with this parameter, MAPLE executes interactively the procedures, asking the user for different situations throughout the computation. This feature has been succesfully used, from the educational point of view.

You may download the MAPLE code from http://www.matesco.unican.es/\%7Eolazabal/software/maples/algelin/english/ casla.zip

Next you must name the downloaded fild 1 and read it over MAPLE. If you compute with MAPLE 7, in order that function splits works it is necessary to include previously the command

$>$ with(polytools):

$\overline{1}$ I suggest "casla" or "psalmo" 
The following in this paper consider the differents sections of the package CASLA in relation to different topics in Linear Algebra: Equivalent, similar and congruent matrices

\section{Procedures for Equivalent Matrices}

Recall that two matrices are equivalent iff they have the same rank; so the basic topic covered is the above mentioned computation of the rank of any matrix over the symbolic field $F=K\left(x_{1}, \ldots, x_{n}\right)$, where $K=\mathbf{Q}$ or the prime field $\mathbf{Z}_{p}$. Computation is made by elementary operations in the rows of the matrix, so Echelon matrices are previously considered; it is straightforward to obtain the LU decomposition. Finally, all is ready to consider linear systems over $F$.

\subsection{Main Procedures}

There are several procedures for this topic; for the sake of simplicity and shortness reasons we will expose only procedures to compute the LU decomposition of any matrix and the procedure for linear systems $A X=B$.

For examples with function RANK, please visit the url:

http://www.matesco.unican.es/\%7Eolazabal/software/maples/algelin/

english/equivalence/rank/rank.html

\section{LU Decomposition}

Function LU may be called by means of:

- LU(A)

- LU(A,p)

Returns the LU decomposition of any matrix

- LU(A,inter)

This command also works over any characteristic. Its interest may be seen in figure 3 .

For another examples, please visit the url:

http://www.matesco.unican.es/\%7Eolazabal/software/maples/algelin/ english/equivalence/LU/LU.html

\section{Linear Systems}

CASLA solves linear systems by means of one of

- $\operatorname{GAUSS}(\mathrm{A}, \mathrm{B})$

in honour to the egregious mathematicien.

- $\operatorname{GAUSS}(\mathrm{A}, \mathrm{B}, \mathrm{p})$

- GAUSS(A,B,unknown)

- GAUSS(A,B,unknown,p)

- GAUSS(A,B,inter)

where A and B must be matrices with the same amount of rows. 


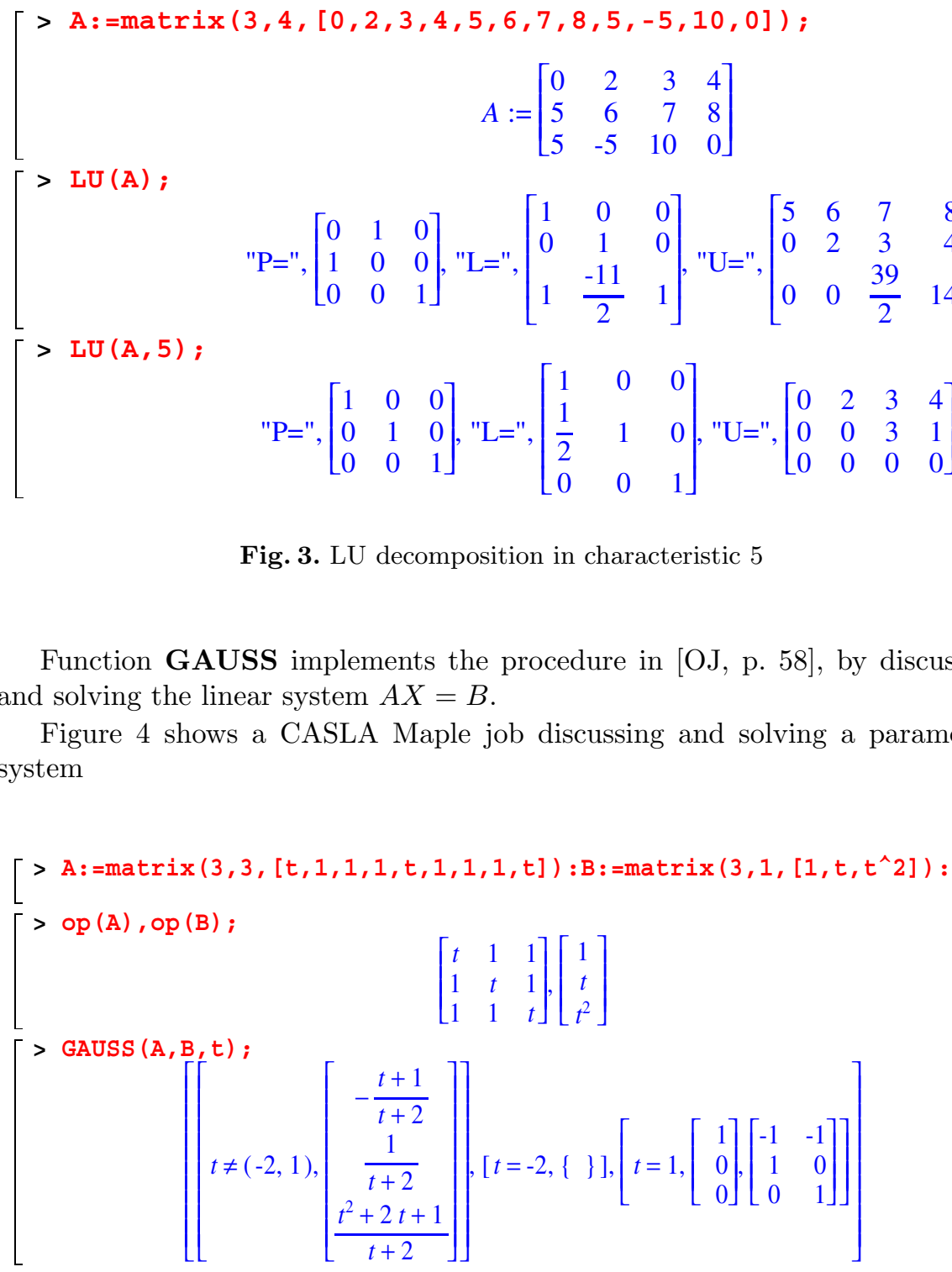

Fig. 4. Parametric systems

For another examples, please visit the url:

http://www.matesco.unican.es/\%7Eolazabal/software/maples/algelin/ english/equivalence/ Systems/systems.html 


\section{Procedures for Similar Matrices}

Recall that two square matrices $A$ and $B$ are similar iff ther exists an invertible matrix $P$ such that $B=P A P^{-1}$. So $\operatorname{det}(x I-A)$ is an invariant for similar matrices, being its roots the known eigenvalues and for each $t, \operatorname{ker}(t I-A)$ is the subspace of eigenvectors. Moreover, it is well known that for any square matrix $A$ there exists $P, R, S$ regular matrices such that

- $F=P^{-1} A P$ is a diagonal-block of companion matrices $C\left(f_{i}\right)$, where $f_{i}$ are the called invariant polynomials of the class. $F$ is called the Rational or Frobenius form.

- $G=R^{-1} A R$ is a diagonal-block of companion matrices $C\left(g_{i}\right)$, where the $g_{i}$ are a power of irreducible polynomials. $G$ is called the Irreducible form.

- If $\operatorname{det}(x I-A)$ splits, $J=S^{-1} A S$ is the known Jordan form of the class.

Several procedures to compute $P, R, S$ and $F, G, J$ build this part of the package. About its new features we remember of the introduction that they work on any characteristic and, for educational purpose, they may be interactively runned.

\subsection{Main Procedures}

\section{Eigenvalues It computes the eigenvalues of $A$}

CASLA computes eigenvalues, over different extensions of the basis field, by means of :

- EIGENVALUES(A)

- EIGENVALUES(A, K)

- EIGENVALUES(A, cerrado)

- EIGENVALUES(A, p, cerrado) computation in the field $\mathbf{Q}$ computation in the field $\mathrm{K}$ in Spanish "closed", computation in $\mathbf{C}$ computation in the closure of $\mathbf{Z}_{p}$

For efficiency reasons, EIGENVALUES(A, cerrado) calls linalg[eigenvalues] of MAPLE. In the other cases, function EIGENVALUES returns a list of pairs

$$
\left[\left[t_{1}, m_{1}\right], \ldots,\left[t_{2}, m_{2}\right]\right]
$$

where the $t_{i}$ 's are the different eigenvalues and $m_{i}$ their multiplicity in the characteristic polynomial.

For examples, please visit the url:

http://www.matesco.unican.es/\%7Eolazabal/software/maples/algelin/ english/similarity/autov/autov1.html 


\section{Eigenvectors It computes the eigenvectors of $\boldsymbol{A}$}

CASLA computes eigenvectors, over different extensions of the basis field, by means of :

- EIGENVECTS(A)

- EIGENVECTS(t,A)

- EIGENVECTS(t,A,K)

(note the UPPERCASES)

where $\mathrm{t}$ is a candidate to eigenvalue and $\mathrm{K}$ any field in Maple sintaxys.

Figure 5] shows a jop computing the eigenvalues and corresponding eigenvectors of the same matrix on different fields

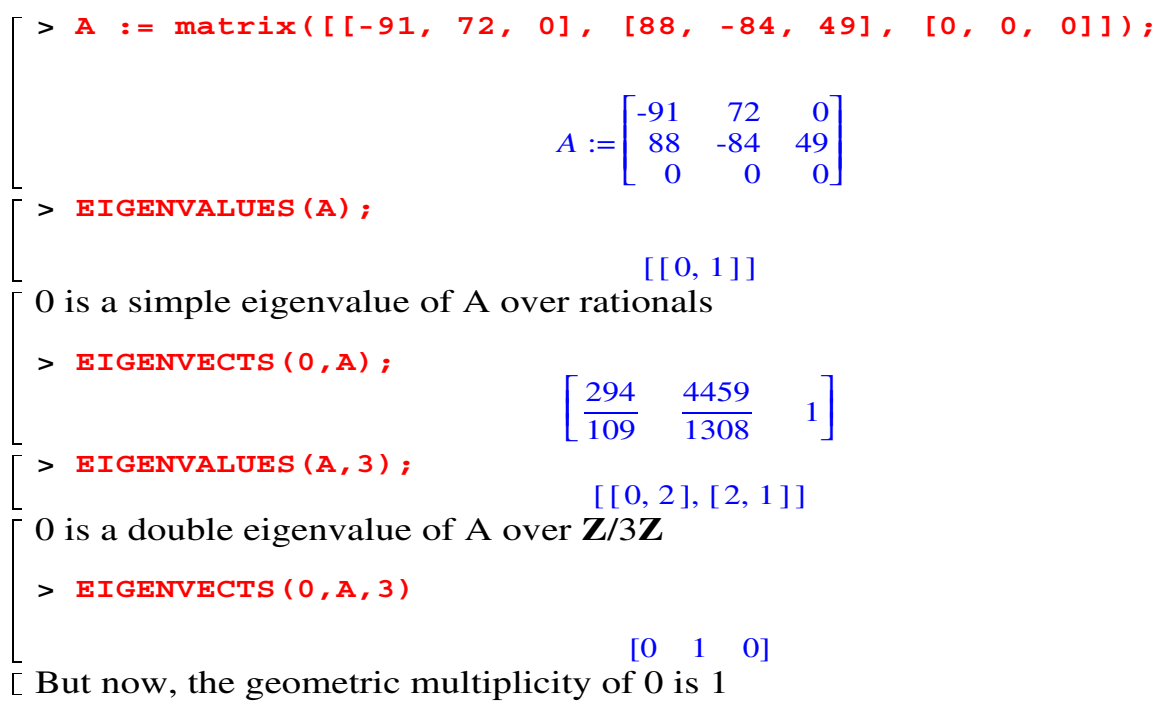

Fig. 5. Eigenvalues and eigenvectors over different characteristic

More examples visiting the url:

http://www.matesco.unican.es/\%7Eolazabal/software/maples/algelin/ english/similarity/autov/autov1.html

\section{Minimum Polynomial It computes the minimumpolynomial of $A$}

CASLA computes the minimum polynomial of a matrix by means of :

- MINIMUM(A,unknown)

- MINIMUM(A, q, unknown) perhaps different from the MINIMUM mod p

- MINIMUM(A, P, unknown)

- $\operatorname{MiNIMUM}(\mathrm{A}, \mathrm{P}, \mathrm{q}$, unknown)

- MINIMUM(A, inter)

where $\mathrm{q}$ is a prime number, and $\mathrm{P}$ an unbounded symbol different from inter, to allocate the transformation matrix. 
Function MINIMUM implements the procedure in [OJ, p. 99], computing a matrix $M=\left(\begin{array}{cc}C(p) & 0 \\ 0 & G\end{array}\right)$ similar to the input matrix, where $p$ is its minimum polynomial. If $A=\left(a_{i j}\right)$

- MINIMUM(A,unknown) returns $p$ (unknown) of $A$, computing in the extension field $\mathbf{Q}\left(a_{i j}\right)$.

- MINIMUM(A, P, unknown) returns the minimum polynomial and it allocates in $P$ the regular matrix such that $P^{-1} A P=M$. It may be asked by means evalm $(\mathrm{P})$ or $\mathrm{op}(\mathrm{P})$. This is a new feature.

For examples, please visit the url:

http://www.matesco.unican.es/\%7Eolazabal/software/maples/algelin /english/similarity/frobenius/frobenius.html

\section{Rational or Frobenius Form}

For $A$ it computes $P, F$ with $P^{-1} A P=F$ the Rational form

There are several possibilities for input the command which compute Frobenius form:

- FROBENIUS(A)

- FROBENIUS(A,P)

- FROBENIUS(A, p)

- FROBENIUS(A, P, p)

- FROBENIUS(A, inter)

where the parameters have the same meaning as above.

Function FROBENIUS implements the procedure in ([OJ], p. 100), computing the (only one) matrix $F=\operatorname{diag}\left[C\left(f_{1}\right), \ldots, C\left(f_{r}\right)\right]$ similar to the input matrix, where $f_{r}|\cdots| f_{1}$, and it allocates in $P$ the transformation matrix.

For examples, please visit the url:

http://www.matesco.unican.es/\%7Eolazabal/software/maples/algelin

/english/similarity/frobenius/frobenius.html

Irreducible Form. This is all new to MAPLE

For $A$ it computes $R, G$ with $R^{-1} A R=G$ the irreducible form This function may be called by means of:

- IRREDUCIBLE(A)

- IRREDUCIBLE(A, K)

- IRREDUCIBLE(A, P)

- IRREDUCIBLE $(\mathrm{A}, \mathrm{P}, \mathrm{K})$

- IRREDUCIBLE(A, inter)

where the parameters are as usually in CASLA. 
Function IRREDUCIBLE implements the procedures in [OJ, p. 116], computing, for a matrix $A$, a matrix $G=\operatorname{diag}\left[C\left(p_{1}\right), \ldots, C\left(p_{r}\right)\right]$ similar to the input matrix, where the $C\left(p_{i}\right)$ are the companion matrices of the elementary divisors of $A$. Moreover it allocates in $P$ a regular matrix such that $P^{-1} A P=G$. If $A=\left(a_{i j}\right)$.

- IRREDUCIBLE(A) and IRREDUCIBLE(A,P) compute in the extension field $\mathbf{Q}\left(a_{i j}\right)$.

- If $\mathrm{K}=\left\{\alpha_{1}, \ldots, \alpha_{n}\right\}$ is a set of algebraic numbers, the procedures IRRE$\operatorname{DUCIBLE}(\mathrm{A}, \mathrm{K})$ and IRREDUCIBLE $(\mathrm{A}, \mathrm{P}, \mathrm{K})$ compute in the extension field $\mathbf{Q}\left(a_{i j}, \alpha_{1}, \ldots, \alpha_{n}\right)$.

- As usually in the package, IRREDUCIBLE(A, inter) runs the procedure interactively, asking the user for different situations throughout the computation, specially with educational purpose.

Next figure 6] shows a CASLA Maple job, computing the irreducible form of a matrix over an appropriate extension of the rationals.

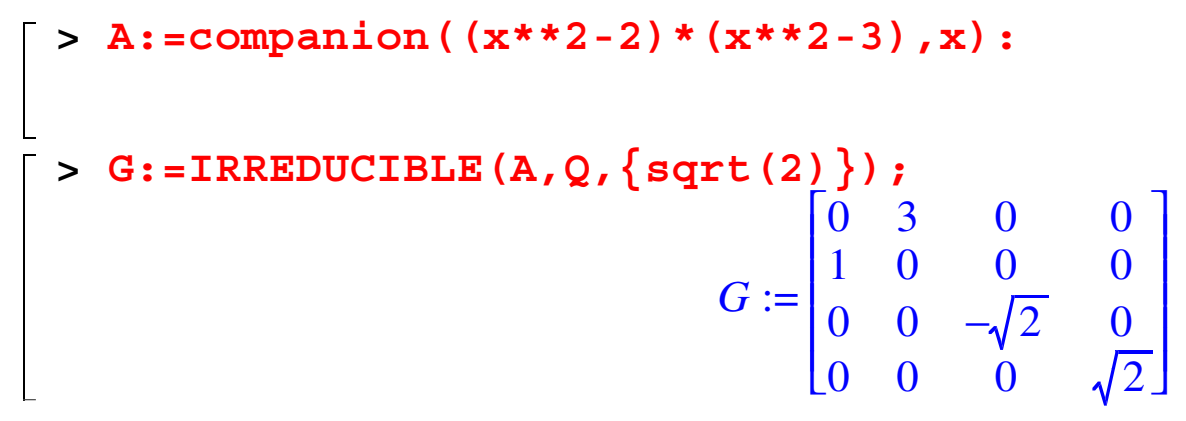

Fig. 6. Irreducible form of a matrix

For another examples, please visit the url:

http://www.matesco.unican.es/\%7Eolazabal/software/maples/algelin /english/Jordan/irreducible/irredu.html

Jordan Form. For $A$ it computes $S, J$ with $S^{-1} A S=J$ the Jordan form

Casla computes the Jordan form of a matrix $A$ as Maple $\mathrm{V}$; i.e., first it computes a cyclid 2 form $C$ of $A$ and after the Jordan form $J$ of $C$, (see [OJ, pp. 121, 123], and [OJ2]).

As usually in CASLA user may choose among different parameters:

$-\operatorname{JORDAN}(\mathrm{A})$

(note the UPPERCASES)

$-\operatorname{JORDAN}(\mathrm{A}, \mathrm{p})$

${ }^{2}$ a similar diagonal-block of companion matrices 
$-\operatorname{JORDAN}(\mathrm{A}, \mathrm{P})$

- $\operatorname{JORDAN}(\mathrm{A}, \mathrm{P}, \mathrm{p})$

- JORDAN(A, inter)

where $\mathrm{p}$ is a prime number in order to work on finite characteristic and $\mathrm{P}$ an unbounded symbol to allocate the matrix transformation.

Next figure 7 shows a small interactive job, asking the user for different situations throughout the computation, specially with educational purpose.

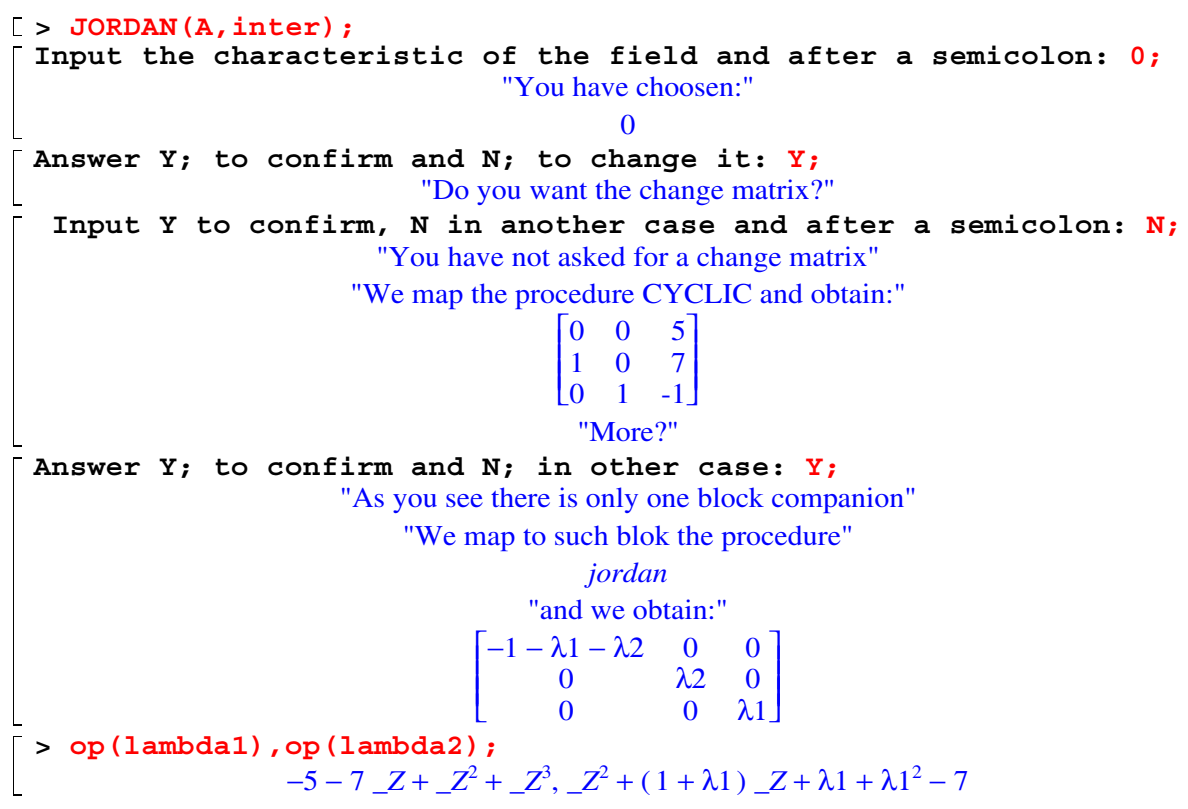

Fig. 7. An interactive job for Jordan form

For another examples, please visit the url:

http://www.matesco.unican.es/\%7Eolazabal/software/maples/algelin /english/Jordan/jordan/jordan.html

\section{Procedures for Congruent Matrices}

In order to avoid a tedious exposition, we are going to set the main procedures:

DIAGONAL: It computes a diagonal congruent to any symmetric matrix over rationals or $\mathbf{Z}_{p}$. For hermitian matrices it computes over gaussian numbers.

CHOLESKY: Over $\mathbf{Q}, \mathbf{Z}_{p}$ or gaussian numbers returns the Cholesky decomposition of any symmetric (hermitian) matrix.

GRAM_SCHMIDT: Computes a basis ortonormal. It allows a positive definite matrix as a metric. 
QR: Computes over reals or complex numbers the QR decomposition. DVS: Computes over reals or complex numbers the DVS decomposition.

For a more complete explanation and examples, please, visit the url: http://www.matesco.unican.es/\%7Eolazabal/software/maples/algelin/ english/index.html

\section{Educational Experience}

At the end of the last academic course, I have surveyed my students about different aspects of learning Linear Algebra. Every student had to choose from 1 to 6 its conformity with the assertion. One of them was:

Using CASLA package has been very useful to learn Linear Algebra.

$18 \%$ of students have choose the answer $4,54 \%$ have choose 5 and $28 \%$ have marked 6 . So, I think the experience was very positive.

\section{References}

[D] Danilevski, A.: On a numerical solution of Vekua's equation . Mat. Sb. 2 (Russian) (1937) 169-171.

[GF] Gantmacher, F.R.: Theory of Matrices I. Chelsea Publishing Company. New York (1977).

[MB] Monagan M.B. et al: MAPLE V. programming Guide. Springer. New York (1996).

[ML] Mulders T.M.L., Levelt, A.H.M.: A package for the computation of several matrix normal forms. Share Library. MAPLE V (1993).

[OJ] Olazábal, J.M.: Procedimientos simbólicos en Álgebra Lineal . Servicio de Publicaciones de la Universidad de Cantabria. Santander. SPAIN (1998).

[OJ2] Olazábal, J.M.: A unified method to determine the canonical forms of any matrix. SIGSAM Bulletin, Issue 127, 33, number 1, (1999), 6-20. 